\title{
GESTÃO DA INOVAÇÃO EM UM INSTITUTO PRIVADO DE P\&D BRASILEIRO: UM ESTUDO DE CAMPO NO ITEMM
}

\author{
INNOVATION MANAGEMENT IN A PRIVATE BRAZILIAN R\&D INSTITUTE: A \\ FIELD STUDY AT ITEMM
}

\author{
Josia de Sousa Santos Filho \\ Especialista em Gestão de Projetos pela PUC MINAS \\ josia.sousa02@gmail.com \\ Sueli Menelau \\ Doutora em Administração pela UnB \\ Professora na Universidade Federal de Pernambuco, \\ Caruaru, Pernambuco - Brasil. \\ suelimenelau@gmail.com \\ Patrícia Lacerda de Carvalho \\ Doutoranda em Administração pela UFPB \\ Professora Substituta na Universidade Federal da Paraíba, \\ João Pessoa, Paraíba - Brasil \\ patricialacerdac@gmail.com \\ Francisco Guilherme Lima Macedo \\ Doutorando em Administração pela UnB \\ Professor do Instituto Superior de Ciências Policiais (ISCP/PMDF) \\ Brasília, Distrito Federal - Brasil \\ guilhermelima03@gmail.com
}

Resumo: Os institutos de Pesquisa e Desenvolvimento (P\&D) são agentes de inovação nos quais a disseminação de tecnologias advindas deles contribui para o direcionamento de organizações e da economia. Com isso, sua gestão da inovação torna-se um recurso organizacional complexo que abrange questões tecnológicas, econômicas e sociais. A pesquisa objetivou analisar a influência da gestão da inovação, das atividades de P\&D, na competitividade de um instituto de pesquisa privado, com sede em diferentes unidades federativas brasileiras. O método, dedutivo com abordagem qualitativa foi executado por meio de estratégias bibliográfica e de campo, e os dados foram analisados por meio de análise de conteúdo. Os sujeitos foram escolhidos entre os funcionários do setor de P\&D do Instituto de Tecnologia Edson Mororó Moura (ITEMM), que busca sempre ofertar às organizações-clientes inovações tecnológicas enquadradas em mais de uma área prioritária da Indústria 4.0, configurando-o como um instituto de vanguarda tecnológica. Os resultados evidenciaram que as principais dificuldades à gestão do $\mathrm{P} \& \mathrm{D}$ do ITEMM são: dependência externa na prototipagem; mudança de escopo nos projetos; distância de outros centros de pesquisa; e infraestrutura. Por sua vez, os facilitadores para o gerenciamento da inovação são: recursos financeiros; equipe multidisciplinar de pesquisa; clientes com experiência em P\&D; e a produtividade não ser avaliada em metas diárias. Conclui-se que a gestão de $\mathrm{P} \& \mathrm{D}$ traz retornos positivos significativos, se configurando como uma estratégia de valor, mas verificou-se que falta ao ITEMM visibilidade na esfera acadêmica de $\mathrm{P} \& \mathrm{D}$, que deve ser buscada para trazer legitimidade ao instituto.

Palavras-chave: Inovação tecnológica. Instituto de pesquisa. Pesquisa e Desenvolvimento.

Abstract: R\&D institutes are agents of innovation in which the dissemination of technologies contributes to the direction of organizations and the economy. With this, its innovation management becomes a complex organizational resource that covers technological, economic, and social issues. The research aimed to analyze the influence of innovation management, R\&D activities, on the competitiveness of a private research institute, headquartered in different Brazilian federative units. The method, deductive with a qualitative approach, was executed through bibliographic and field strategies, and the data were analyzed through content analysis. The subjects were chosen from the employees of the R\&D sector of Instituto de Tecnologia Edson Mororó Moura (ITEMM), which always seeks to offer client organizations technological innovations framed in more than one priority area of Industry 4.0, configuring it as a cutting-edge technological institute. The results showed that the main difficulties in managing ITEMM R\&D are: externa dependence on prototyping; change of scope in projects; distance from other research centers; infrastructure. In turn, the facilitators for innovation management are financial resources; multidisciplinary research team; customers with R\&D experience; and productivity not be measured on daily goals. It is concluded that R\&D management brings significant positive returns, being configured in a value strategy, but it was found that ITEMM lacks visibility in the academic sphere of $\mathrm{R} \& \mathrm{D}$, which must be sought to bring legitimacy to the institute.

Keywords: Technological innovation. Research institute. Research and Development.

Cite como

American Psychological Association (APA)

Santos Filho, J. S., Menelau, S., Carvalho, P. L., \& Macedo, F. G. L. (2021, jul./dez.). Gestão da inovação em um instituto privado de P\&D brasileiro: um estudo de campo no ITEMM. Revista Inovação, Projetos e Tecnologias - IPTEC, São Paulo, 9(2), 197-215.

https://doi.org/10.5585/iptec.v9i2.19419. 


\section{Introdução}

O desenvolvimento econômico de um país está diretamente relacionado ao progresso técnico, sendo aquele resultado do desdobramento da sociedade, de seus conhecimentos e de suas instituições (Mazzucato, 2014; Tunes, 2015). Esse contexto evidencia que o êxito na competição global do século XXI está subordinado ao desenvolvimento de produtos que sejam inovadores e que oportunizem uma resposta rápida às empresas (Arcuri, 2016). O ponto crítico é que o gerenciamento da atividade inovativa de uma organização deve estar fundamentalmente alinhado aos seus objetivos, assim como ao posicionamento de mercado por ela adotado (Bin et al., 2015; Findik \& Beyhan, 2015).

Em nações da União Europeia, em algumas da Ásia e nos Estados Unidos, a inovação é oportunizada por organizações privadas com recursos tecnológicos que contribuem para seu desenvolvimento, apoiadas por programas públicos de financiamento sustentável (Mazzucato, 2014). Em países como o Brasil, a participação do Estado está relacionada ao fomento de pesquisas acadêmicas, sendo proporcionalmente maior em relação a outros países (Verma et al., 2011). A capacidade limitada de evolução tecnológica do Brasil incorre na baixa participação na produção de bens de capital, visto que a disseminação de tecnologia de origem industrial para outros setores é pouco expressiva (Mazzucato, 2014; Menelau et al., 2019). Ratifica essa informação o relatório do Fórum Econômico Mundial de 2016, que aponta que o país ocupa a posição $72^{\mathrm{a}}$ no ranking que mede o desenvolvimento tecnológico das nações (Baller et al., 2016).

Destaca-se que mudanças tecnológicas surgem a partir de atividades inovadoras, incluindo-se nesse grupo investimentos intangíveis, como em Pesquisa e Desenvolvimento $(\mathrm{P} \& \mathrm{D})$, que proporcionam à organização uma capacidade maior para investida na produção, a qual constituirá mais empregos e outras rendas (Arcuri, 2016; Organização para a Cooperação e Desenvolvimento Econômico [OCDE], 1997). As atividades de P\&D das empresas almejam obter a inovação tecnológica, e as organizações, ao utilizarem-se das tecnologias existentes para seu desenvolvimento, elevam seu grau de importância, passando a inovação a ter participação significativa nas estratégias organizacionais, aumentando sua competitividade (Campos et al., 2017; Govindarajan \& Trimbre, 2006).

Com isso em vista, os institutos de $\mathrm{P} \& \mathrm{D}$ tornam-se essenciais para processos inovativos e destacam-se por seu papel e suas técnicas no desenvolvimento do ramo científico e tecnológico, além de se fazerem presentes no contexto econômico e social de países (Hales, 2001; Sperotto \& Tartaruga, 2017; Veado, 1985). Os institutos de P\&D, portanto, contribuem 
para o desenvolvimento e a manutenção de economias nacionais através da interação com a indústria e com sistemas de inovação, ao proporcionarem serviços técnicos para o fomento de suas atividades inovadoras (Albert et al., 2016; Veado, 1985). Ademais, institutos de P\&D são agentes de inovação aplicáveis tanto em ambientes públicos como privados, representando seus interesses e suas ações (Tunes, 2015; Veado, 1985).

A partir dessas premissas, é possível asseverar que a inovação e a disseminação de tecnologias, estimuladas por investimentos em $P \& D$ e por capacitação técnica, contribuem para o direcionamento de empresas e, consequentemente, das economias nacionais para um crescimento mais sustentável (Verma et al., 2011). Ainda assim, o que se observa no Brasil é que a geração de novas tecnologias que dinamizem sua economia nacional é incipiente, e que o processo de catching up, ao longo do tempo, depende da convergência da inovação com sua gestão, principalmente, mas não apenas, nas organizações (Arcuri, 2016; Rauen, 2016).

Nesse sentido, deve-se buscar fomentar ferramentas de gestão que criem condições para que atividades de $\mathrm{P} \& \mathrm{D}$ oportunizadas nos institutos de $\mathrm{P} \& \mathrm{D}$ aumentem a produção e fortaleçam a conjuntura econômica (Arcuri, 2016; Rauen, 2016). A opção por se estudar institutos de P\&D, portanto, justifica-se pela possibilidade de se entender melhor a relação que existe entre $P \& D$ e gestão organizacional, uma vez que esses institutos, na medida em que oferecem suporte fundamental ao alcance da inovação - desde sua fase inicial até sua introdução no mercado contribuem para seu desenvolvimento, atraindo novos clientes, vencendo desafios e oportunizando destaque e visibilidade com suas descobertas no mercado.

Tendo em vista a relevância do assunto, em busca realizada no Portal de Periódicos da Coordenação de Aperfeiçoamento de Pessoal de Nível Superior (Capes) em julho de 2020, empregando como termos de busca "instituto de pesquisa", "instituto de P\&D", "instituto de pesquisa e desenvolvimento" e "instituto e centro de pesquisa" - grafando-se instituto no singular e no plural, nos campos título e assunto -, verificou-se uma baixa produção em português de artigos em periódicos revisados por pares, com o resgate de 15 textos, sendo o mais recente de 2015. A escolha de realização desta pesquisa em um instituto de P\&D se deu tanto diante desse achado quanto do escopo delineado. Desse modo, o objetivo desta pesquisa foi analisar a influência da gestão da inovação, das atividades de P\&D, na competitividade de um instituto de pesquisa privado, com sede em diferentes unidades federativas brasileiras.

Avalia-se que as justificativas teórica e empírica deste estudo residem na premissa de que esta análise poderá contribuir ao entendimento dos institutos privados de $\mathrm{P} \& \mathrm{D}$ brasileiros - organizações do Sistema Nacional de Inovação (SNI) menos estudadas em comparação aos institutos públicos -, elementos essenciais à economia da inovação e ao progresso tecnológico, 
frente ao declínio dos principais indicadores agregados de inovação no país, entre eles, a redução de investimentos em P\&D (Instituto Brasileiro de Geografia e Estatística [IBGE], 2017; Menelau et al., 2019; Zanello et al., 2016).

\section{Fundamentação teórica}

Inovação pode ser compreendida como uma prática, uma ideia ou um objeto identificado como uma novidade, que pode vir a ser utilizada pela organização em um determinado processo (Rogers \& Shoemaker, 1971; Tunes, 2015). Inovação frequentemente é associada à mudança, referindo-se, quando relacionada a uma organização, a sua capacidade de adaptação, emergindo reativamente ao ambiente externo ou de maneira preventiva a ele (Campos et al., 2017; Damanpour, 1996). Na tipologia mais difundida, inovação é identificada como a introdução de um novo produto (e ou serviço), de um novo processo que apresente uma melhoria na qualidade, de um novo método de marketing, ou de um novo modelo organizacional significativamente melhorado e perceptível para o cliente (OCDE, 1997; Schumpeter, 1988).

Diferentes graus das inovações classificam-nas, quanto a sua abrangência, em radicais ou incrementais (Schumpeter, 1988). A inovação radical considera a introdução de um produto, um processo ou até mesmo um serviço inteiramente novo, trazendo consigo uma mudança tecnológica; a incremental, por seu turno, refere-se às pequenas diferenças sobre outra inovação, introduzidas aos poucos nas rotinas organizacionais (Damanpour, 1991; Dosi, 1998). Apesar de no Brasil boa parte das inovações serem incrementais, é possível encontrar inovações de impacto abrangente - ainda que, com alcance mundial, sejam raras - nas classificadas como estritamente tecnológicas e nas atividades industriais (Sperotto \& Tartaruga, 2017).

Para ocorrer incrementos nos processos tecnológicos, faz-se necessária a junção entre conhecimento científico e inovações, acarretando o desenvolvimento de produtos e procedimentos que anteriormente estavam no nível de pesquisa básica (Albert et al., 2016; Dosi, 1998). Assim, compreender a inovação tecnológica em uma organização implica buscar a melhoria contínua, englobando recursos físicos, humanos e tecnologias (Findik \& Beyhan, 2015; Gonçalves et al., 2006). Esses pontos destacam-se como relevantes e satisfatórios ao alcance de inovação, uma vez que constroem estratégias internas para o desenvolvimento de capacidades e avanço na execução de P\&D, conhecidas como catching-up (Bin et al., 2015).

A gestão da inovação é entendida como um recurso organizacional que precisa estar inserido nas estratégias empresariais (Campos et al., 2017; Zanello et al., 2016), e associa-se à estruturação de uma abordagem baseada em objetivos específicos, planejamento claro e 
levantamento de informações, buscando a solução de possíveis problemas ligados à estrutura gerencial (Bessant \& Tidd, 2009; Tushman et al., 1997). E ainda que haja uma pluralidade de abordagens, a gestão da inovação deve refletir a composição de processos organizacionais centrais e subjacentes - pelos quais a inovação deve ser conduzida (Silva et al., 2014).

Acrescenta-se que a gestão da inovação não surge por um acaso, e sim de conhecimentos que são estimulados e atrelados às estratégias organizacionais, devendo ainda adequar-se ao contexto organizacional (Bessant \& Tidd, 2009). A gestão da inovação precisa ser disseminada na organização e contar com a chefia, uma vez que a liderança é sua facilitadora (Rice et al., 2008; Simantob \& Lippi, 2003). Considera-se a gestão da inovação um processo complexo que abrange questões tecnológicas, econômicas e sociais, capazes de influenciar os ambientes interno e externo da organização (Bessant \& Tidd, 2009; Campos et al., 2017). E, pondera-se que a gestão da inovação em organizações que geram tecnologia precisa ser entendida tanto pelos complexos parâmetros organizacionais que a suportam (Silva et al., 2014) quanto pelo contexto institucional em que está inserida.

Nesse sentido, quando a gestão da inovação passa a ser observada para além das fronteiras organizacionais, compreende-se o escopo de um SNI com relações e organizações que a operacionalizam em nível institucional em um determinado país. Com isso, os esforços de fortalecimento da gestão da inovação e de enfrentamento dos desafios de se gerenciar laboratórios e centros de $\mathrm{P} \& \mathrm{D}$ nas empresas - sejam públicas ou privadas - extrapolam o aperfeiçoamento do SNI, em busca de se alcançar integração e soluções singulares de Ciência, Tecnologia e Inovação (CT\&I) (Arcuri, 2016).

Quanto ao Brasil, comparando-se ao cenário internacional, a gestão da inovação das organizações que compõem o SNI é impactada pelo protagonismo estatal no financiamento, na quantidade excessiva de instalações pequenas de $P \& D$ e na menor frequência de doutores trabalhando nessas estruturas (Arcuri, 2016; Sperotto \& Tartaruga, 2017). Ainda assim, o conjunto de agentes que interagem para a efetivação da P\&D do SNI objetivam expandir o estoque existente de conhecimentos científico e tecnológico de organizações, a aplicação desses conhecimentos e sua consequente comercialização (Campos et al., 2017; OCDE, 1997).

Especificamente sobre a finalidade da $\mathrm{P} \& \mathrm{D}$, essa atividade, em um instituto público ou privado, objetiva a criação de conhecimento e a capacitação dos indivíduos que participam da sua formação, e suas ações trazem benefícios à organização, independentemente do alcance da finalidade inicial, excedendo o delineamento da inovação (Hales, 2001; Hasegawa \& Furtado, 2006). Em um SNI, cabem aos institutos de P\&D, públicos e privados (Figura 1): 


\section{Figura 1.}

Funções de Institutos de $P \& D$
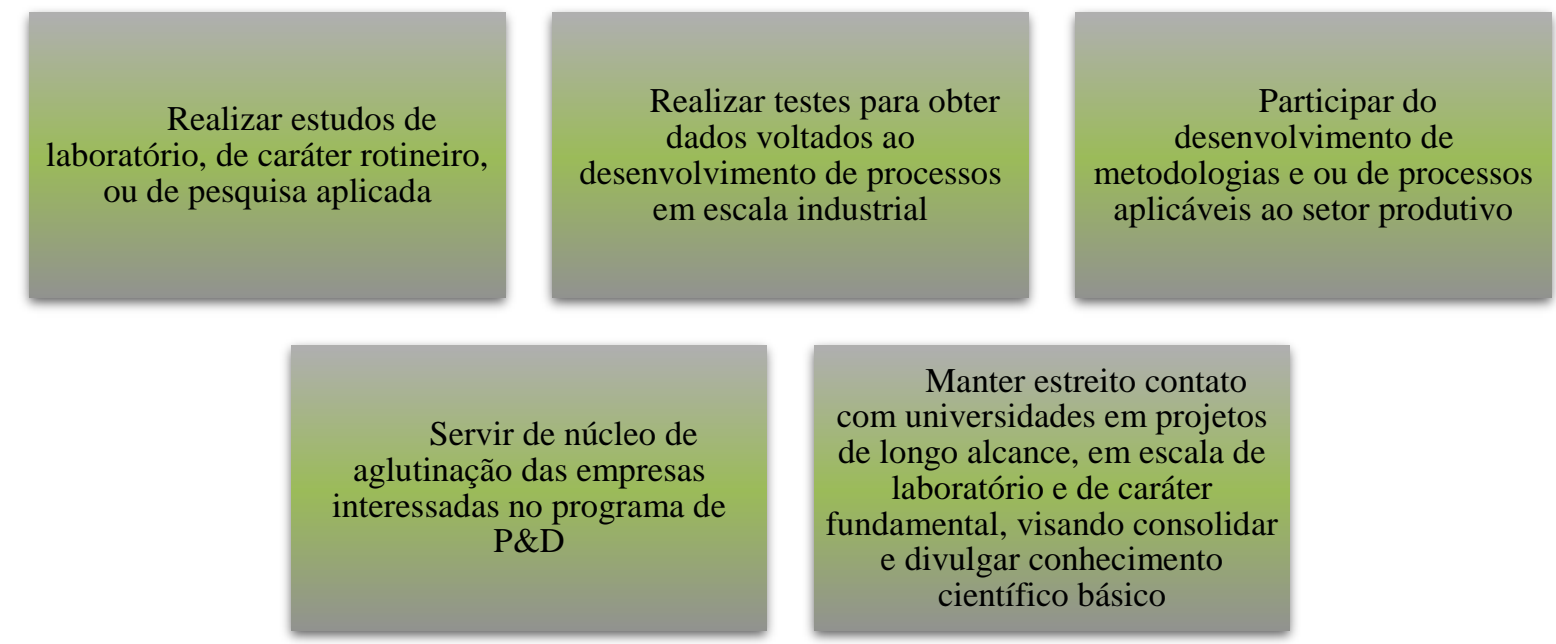

Fonte: Adaptado de Veado (1985).

Direta ou indiretamente, governos incentivam as funções dos institutos de P\&D públicos ou privados (Figura 1), uma vez que entendem que elas são essenciais ao desenvolvimento econômico de um país pelo potencial aumento da produtividade em áreas distintas, como saúde, agricultura, bens de consumo, entre outros (Albert et al., 2016; Arcuri, 2016; Hales, 2001; Mazzucato, 2014). O fato é que vem se fortalecendo a premissa de que se faz necessário desenvolver uma estratégia para os Estados nacionais fundamentada em inovação, enfatizando a gestão da inovação dos entes do SNI, apesar das dificuldades (Freeman \& Soete, 1997).

No escopo da empresa, os benefícios dos institutos de P\&D envolvem conhecimentos gerados e experiências adquiridas durante a criação e o desenvolvimento de um projeto de inovação, que precisam ser aplicados em outros produtos ou processos, oportunizando resultados distintos dos planejados (Findik \& Beyhan, 2015; Hasegawa \& Furtado, 2006; Sperotto \& Tartaruga, 2017).

\section{Metodologia}

O método da pesquisa é dedutivo, a abordagem utilizada foi a qualitativa, e se estabeleceu dois objetivos metodológicos: descritivo e exploratório (Bogdan \& Biklen, 2003). Quanto à temporalidade, é um estudo transversal. As estratégias de pesquisa empregadas foram duas: bibliográfica - para o referencial que lastreia o estudo e auxiliar no entendimento das categorias -; e de campo, para o fenômeno estudado nos dados primários (Creswell, 2007).

O lócus do estudo foi o Instituto de Tecnologia Edson Mororó Moura (ITEMM), entidade sem fins lucrativos dedicada a projetos de P\&D e inovação de baterias de chumbo 
ácido. O ITEMM possui três unidades, sendo duas em Pernambuco - em Recife e em Belo Jardim (sede) - e uma em São Paulo - em São José dos Campos. O setor de P\&D do ITEMM é composto por 31 funcionários das três unidades, distribuídos entre: $(i)$ engenheiros de diversas áreas (eletricistas, eletrônicos, de materiais, mecânicos e químicos); (ii) técnicos eletromecânicos (que operacionalizam testes e experimentos); e (iii) bolsistas de pesquisa (em nível de mestrado e doutorado).

Os entrevistados foram definidos entre os funcionários do setor de P\&D do ITEMM, pois avaliou-se que seriam os indivíduos com conhecimento suficiente sobre as características do fenômeno (Creswell, 2007). Seis sujeitos aceitaram participar, identificados como E1 a E6. O instrumento utilizado para coleta de dados primários foi um roteiro de entrevista semiestruturado, elaborado pelos pesquisadores, com 18 perguntas distribuídas em três partes, a saber: uma breve descrição do estudo e de seu objetivo e o informe de participação voluntária; em sequência, perguntas fundamentadas nas categorias buscadas (ver Tabela 1); e, por fim, questionamentos sobre o perfil socioeconômico.

\section{Tabela 1.}

Categorias de Análise

\begin{tabular}{|c|c|c|}
\hline $\begin{array}{l}\text { CATEG } \\
\text { ORIA }\end{array}$ & CONCEITO & OBJETIVO \\
\hline Inovação & $\begin{array}{l}\text { Está relacionada à mudança, referindo-se à } \\
\text { capacidade de adaptação da organização; inovações } \\
\text { surgem em resposta à modificação do ambiente externo } \\
\text { ou de maneira preventiva a ele (Damanpour, 1996) }\end{array}$ & $\begin{array}{l}\text { Identificar aspectos gerais } \\
\text { sobre as inovações desenvolvidas } \\
\text { pelas atividades de P\&D do } \\
\text { ITEMM }\end{array}$ \\
\hline $\begin{array}{l}\text { Gestão da } \\
\text { inovação }\end{array}$ & $\begin{array}{l}\text { É assegurada por um sistema gerencial que permite } \\
\text { que a organização inove ordenadamente, objetivando } \\
\text { sua sobrevivência e o aumento da competividade } \\
\text { organizacional (Rice et al., 2008; Silva et al., 2014) }\end{array}$ & $\begin{array}{l}\text { Detectar características da } \\
\text { gestão da inovação que aumentam } \\
\text { (ou diminuem) a competitividade } \\
\text { do ITEMM }\end{array}$ \\
\hline P\&D & $\begin{array}{l}\text { Considera-se que a pesquisa é utilizada como } \\
\text { instrumento para se alcançar novos conhecimentos, } \\
\text { enquanto o desenvolvimento se relaciona à aplicação } \\
\text { desse conhecimento com o objetivo de atingir } \\
\text { resultados concretos (Veado, 1985) }\end{array}$ & $\begin{array}{l}\text { Relacionar as atividades de } \\
\text { P\&D do ITEMM como } \\
\text { ferramentas do processo de } \\
\text { inovação }\end{array}$ \\
\hline
\end{tabular}

Fonte: Elaboração própria.

As entrevistas foram ministradas pelos pesquisadores e ocorreram de forma presencial e a distância. Excetuando-se as entrevistas que foram respondidas de maneira escrita, as demais 
foram gravadas, com consentimento dos entrevistados, e duraram de 40 a 50 minutos. As respostas em áudio foram transcritas. A técnica utilizada para exame das entrevistas foi de análise de conteúdo, conforme Bardin (2011), que seguiu os seguintes passos: pré-análise, exploração do material e tratamento dos resultados.

Ressalta-se que, segundo o estabelecido pelas diretrizes éticas para pesquisa científica com seres humanos, o estudo foi desobrigado de registro ou avaliação no Conselho Nacional de Ética em Pesquisa (CNS, 2016). Seguiu-se a conduta de: não ter sido anotada ou solicitada a identificação dos participantes; não ter sido realizada intervenção experimental nos participantes que gerasse riscos acima dos da vida diária; e nenhum diagnóstico ou aconselhamento foi ofertado como consequência das respostas ou com qualquer outra base.

\section{Análise dos resultados}

Fundado em 2012, o ITEMM, a partir de demandas da indústria de baterias de chumbo ácido, busca inovações comprometidas com o meio ambiente e a sustentabilidade. Sua sede apresenta setores que realizam atividades e processos financeiros, de compras, de recursos humanos, de controladoria, de escritório de projetos, de qualidade e outros operacionais do ITEMM. O ambiente de P\&D conta com laboratórios para a pesquisa de ensaios elétricos, eletrônica, prototipagem, x EV e baterias avançadas, e ensaios químicos e materiais.

De acordo com o site institucional (https://www.itemm.org.br/, recuperado em 15, outubro, 2018), a missão do ITEMM é ser o instituto de ciência e tecnologia de referência nacional em soluções de acumulação de energia, investindo em fontes sustentáveis de geração de energia, mobilidade urbana e elétrica, e baterias desenvolvidas, objetivando apresentar esses produtos ao mercado. Avaliou-se que essa concepção comunicada pelo ITEMM está alinhada ao que a literatura identifica como conceito, postura e tarefas desenvolvidas por um instituto de P\&D (ver Hales, 2001; Rauen, 2016; Veado, 1985). O foco de atuação do ITEMM é direcionado à $\mathrm{P} \& \mathrm{D}$ em três áreas: acumuladores de energia avançados, eletrificação veicular, e sistemas de acúmulo de energia e energias renováveis e alternativas.

Os seis entrevistados são engenheiros envolvidos nas atividades de P\&D e inovação, lotados nas três unidades. A maioria dos participantes têm em torno de 30 anos, são homens e casados (apenas uma mulher participou). Os respondentes possuem pós-graduações em diversos níveis em engenharia, e já exerceram algum tipo de atividade anterior de P\&D em outra empresa. Essas informações demonstram que a qualidade da mão de obra está alinhada ao esforço de se influenciar positivamente a capacidade inovadora do instituto (Sperotto \& 
Tartaruga, 2018), e diferenciam positivamente o ITEMM das demais organizações de P\&D que caracterizam o SNI brasileiro, tomando-se como referência Arcuri (2016).

Sobre as categorias visadas para a análise de conteúdo, primeiramente identificou-se aspectos gerais sobre as inovações desenvolvidas. O senso compartilhado pelos seis entrevistados é de que, apesar do foco do ITEMM ser em inovações de tecnologia, a inovação desenvolvida pela P\&D do instituto pode ocorrer de muitas maneiras, seja nos produtos, nos processos que foram significativamente melhorados, assim como na estrutura organizacional ou mesmo nas pessoas, tal qual preconizado por Damanpour (1996) e Schumpeter (1988).

Observa-se que a concepção prioritária de inovação tida pelos entrevistados é de uma ferramenta que adiciona valor, seja na tecnologia em vista seja na organização, conforme Rogers e Shoemaker (1971). Para o E1, “a inovação é [...] agregar valor ao produto, disponibilizar esse produto para o mercado, garantir uma percepção de valor... Para o ITEMM, o termo inovação significa viabilizar produtos". Em concordância, E4 diz que a "inovação é na verdade uma ponte, ela liga um valor de negócio, um valor de mundo em uma realidade atual".

Destacou-se também nas falas que uma das principais características almejada pelos pesquisadores do instituto relacionadas à inovação desenvolvida é o retorno econômico, tal qual em Rieg e Alves Filho (2003) e Schumpeter (1988). No ITEMM, a inovação vem sendo obtida através de características tecnológicas de produtos e processos postos no mercado, impactando significativamente nos retornos da organização e no setor econômico no qual o instituto atua.

Em mais da metade das respostas, também emergiu a concepção de inovação semelhante à proposta por Rogers e Shoemaker (1971), como uma novidade que pode vir a ser utilizada pela organização em um determinado processo, conforme E1, E2 e E6. Já para o E5, complementa-se a essa concepção o entendimento de que o grau da inovação deve ser amplo, institucional e se valer de caráter disruptivo à resolução de problemas nos quais as soluções existentes não (mais) atendem a uma necessidade, como destacado em sequência:

Inovação significa a ruptura do que a gente tem como soluções, a gente atualmente tem problemas em nossa cidade como um todo, e esses problemas eles só são problemas porque as soluções, ditas convencionais, não conseguem resolvê-los, então pra isso é necessário que se encontre novas soluções, e essas novas soluções são inovações [...] O ITEMM se encaixa nisso também. (E5).

Esse pensamento de E5 demonstra o reconhecimento do ITEMM de que suas inovações estejam associadas ao debate atual da contribuição da tecnologia para os anseios gerais da sociedade. E5 também reforça a ideia defendida por Dosi (1998) - e demais autores que 
pesquisam SNIs, a exemplo de Arcuri (2016) e Campos et al. (2017), cuja ênfase se dá nas inovações em produtos e tecnológicas -, que destaca essa forma de inovação como radical.

Com vistas a escrutinar a segunda categoria proposta para a análise, buscou-se detectar características da gestão da inovação que aumentam (ou diminuem) a competitividade do ITEMM. Inicialmente verificou-se se os entrevistados avaliavam que a inovação desenvolvida no ITEMM seria uma resposta ao ambiente externo (elemento reativo) ou seria estímulo do instituto à sociedade (elemento ativo). De acordo com os entrevistados, as atitudes, de ação e reação, se equilibram no ITEMM, como pode ser visto na resposta de E5:

Aqui existem duas linhas de pensamentos, a primeira é de demandas da sociedade todo [...] existe também a geração de soluções que, embora não tenham sido diretamente demandadas pela sociedade, o ITEMM, a partir da concepção digamos sociológica do ambiente que vive e também da do pensamento crítico sobre onde estamos, é capaz de gerar soluções.

E3 e E5 apontaram que a dedicação exclusiva dos funcionários do ITEMM às atividades de P\&D, diferente de quem trabalha em fábricas ou em universidades, constitui um elemento positivo à gestão da inovação. Esse pensamento está afinado com Hales (2001), para quem a contribuição da capacidade de aplicação dos institutos deve ser para habilidades relacionadas à área de P\&D tecnológico, visto ser decisiva para resolução de problemas das indústrias.

Também buscou-se compreender a gestão da inovação, a partir da vivência dos que exercem a atividade de $P \& D$. Nesse sentido, os entrevistados elencaram suas principais dificuldades, assim como os facilitadores, para a ocorrência de P\&D no ITEMM (Tabela 2).

\section{Tabela 2.}

Dificuldades e Facilitadores à Gestão da Inovação no ITEMM

\begin{tabular}{ll}
\hline \multicolumn{1}{c}{ DIFICULDADES } & \multicolumn{1}{c}{ FACILITADORES } \\
\hline Importante dependência externa na prototipagem & Disponibilidade de recursos financeiros \\
Mudança de escopo constante nos projetos & Equipe multidisciplinar \\
Distância de outros centros de pesquisa & Equipes de apoio à pesquisa \\
Falta de infraestrutura (equipamentos) & Clientes com experiência em P\&D \\
Quebrar paradigmas com relação à pesquisa & Produtividade não ser avaliada em metas diárias \\
\hline
\end{tabular}

Nota. As informações não foram organizadas seguindo escore classificatório.

Fonte: Elaboração própria.

Apurou-se na Tabela 2 uma dicotomia: enquanto quatro entrevistados apontaram que recursos financeiros é um facilitador, dois avaliaram que falta infraestrutura à P\&D no ITEMM. E, em que pese a aludida dificuldade ocasionada pela localização da sede - no interior de PE - 
a escolha do ITEMM parece voltada a outros objetivos (que não foram precisados pelos entrevistados), uma vez que a dinâmica espacial de distribuição de hubs de inovação no Brasil é historicamente desfavorável à região Nordeste do país (Tunes, 2015).

Sobre o início do processo de inovação, esse apresenta origens diversas, com demanda identificada pela cúpula estratégica de organizações-clientes e em fóruns técnicos, como também pelo desejo do ITEMM de investir em novas áreas. Verifica-se que essa concepção adotada pelo instituto se alinha às premissas de Schumpeter (1988) e de Morris (2011), no sentido de abertura de um novo mercado e definição de cenário para inovar e fixar o portfólio.

As origens de novos estímulos para inovação no ITEMM, portanto, são diversas, demonstrando que a assimilação de novas fontes de informação para o instituto segue uma tendência de inovação aberta (Chesbrough, 2006). Quando perguntados se metas, objetivos, estratégias - entre outros elementos que configuram um planejamento voltado à competitividade - são traçados na gestão da inovação, os entrevistados informaram sua existência. A resposta de E2 é a que melhor descreve a dinâmica presente no contexto:

A presidência [...] define as diretrizes projetando um cenário de 5 anos para a frente [...] pode haver mudanças, essas revisões são necessárias. Após essas projeções, as metas estratégicas são traçadas pela direção e gerências, e que juntamente com sua equipe realizam o desdobramento dessas metas; nesse processo de desdobramento das diretrizes, ocorre a definição dos projetos para o alcance das estratégias traçadas.

Os entrevistados também afirmaram que a definição estratégica toma por base o seu principal cliente, uma empresa de produção de acumuladores de energia (a Moura). Esse aspecto não é considerado negativo ao desenvolvimento de $\mathrm{P} \& \mathrm{D}$ pelos entrevistados, percepção alinhada com a visão da OCDE (1997) e de Rauen (2016). Isso, porque a busca pelo desenvolvimento de produtos condicionada à demanda e ao conhecimento do cliente, como suporte no desenvolvimento de inovação, contribui para a identificação de novas oportunidades e a relação com fornecedores, como já apontado por Arcuri (2016).

Sobre a participação dos funcionários da definição de metas traçadas com o auxílio dos pesquisadores, "existe [...] colaboração na qual os pesquisadores e o pessoal do administrativo colaboram para definir esses prazos... Existe espaço para que os pesquisadores discutam e é sempre colegiada" (E4). O posicionamento apresentado é relevante à gestão do $\mathrm{P} \& \mathrm{D}$, pois é apontado pela literatura ser necessário apoio da cúpula estratégica de uma organização para que o processo seja facilitado (Findik \& Beyhan, 2015; Rice et al., 2008).

Especificamente sobre o direcionamento estratégico de gestão da inovação no ITEMM: "gaps estratégicos são definidos dentro do ITEMM, [...]; para conseguir isso, a gente acha que 
tem que ir por essa rota e a inovação é assim... Essa meta macro, muitas vezes, é mais engessada, mas o estratégico é como a gente faz o caminho para chegar lá” (E4).

Os entrevistados ressaltaram que a gestão da inovação proporciona desenvolvimento e crescimento sustentável para o ITEMM, como destacado por Rauen (2016), Sperotto e Tartaruga (2017) e Zanello et al. (2016). As atividades de P\&D são o cerne do instituto e de caráter ininterrupto. Nas falas, a sustentabilidade empresarial emerge alinhada à melhoria contínua, como em pesquisas anteriores (e.g., Bessant \& Tidd, 2009; Gonçalves et al., 2006):

[...] os investimentos em $P \& D$ acabam por gerar um círculo que se autoalimenta: investimentos trazem resultados, que geram aumento de competência e conhecimento, que se transformam em reconhecimento e confiança dos clientes, que por sua vez investem e aumentam suas demandas ao instituto. (E3).

No ITEMM, há um foco no desenvolvimento de produtos, o que normalmente demanda uma continuidade nos desenvolvimentos. As práticas usuais dos projetos estão alinhadas com o ciclo de desenvolvimento de solução. (E6).

Apesar da cultura de inovação estabelecida pelo ITEMM incorporar positivamente as diversas mudanças que podem ocorrer em seus projetos, os entrevistados advertem que se faz necessário um trabalho contínuo na gestão da inovação para se desenvolver resultados adversos obtidos, quando convenientes. É importante reiterar que, embora a incerteza seja característica inerente à inovação, superar obstáculos ao trabalho criativo que amplia o conjunto de conhecimentos da organização constitui-se um dos recursos básicos à gestão da inovação (Sperotto \& Tartaruga, 2017). Os entrevistados apontam, ainda, que essa postura da gestão para resultados - sejam positivos ou negativos - advém da confiança do retorno financeiro da P\&D.

A visão transversal que emerge dos entrevistados - temporalidade, trajetória de inovação, inovação em processo e complexidade dos projetos envolvida - alinha-se ao posicionamento da OCDE (1997), que ressalta que a P\&D se refere ao trabalho criativo e objetiva expandir os conhecimentos científicos e tecnológicos existentes, assim como a aplicação diversificada desse conjunto. Soma-se a isto a ideia de inovação aberta e colaborativa - como em Chesbrough (2006) e Findik e Beyhan (2015) - e o combate aos efeitos da empresa ser oriunda de um país emergente (Menelau et al., 2019), contidos nas falas, destacando-se:

O ITEMM conta com uma equipe de consultores externos e, além disso, a direção e membros das equipes de $P \& D$ fazem diversas viagens para centros de pesquisa no Brasil $e$ no exterior. Naturalmente aparecem novidades que, em muitos casos, são logo incorporadas e provocam modificações e ajustes nos direcionamentos estratégicos do instituto. (E3). 
Um elemento ainda a ser apontado é que os entrevistados evidenciam a importância da junção do conhecimento científico com o tecnológico com o objetivo de obter um efeito, como em Veado (1985) e Verma et al. (2011).

No que se refere à terceira categoria analisada - relacionar as atividades de P\&D do ITEMM como ferramentas do processo de inovação -, quando perguntados sobre as atividades de P\&D e o aproveitamento dos resultados, com a identificação de novas tecnologias que possam agregar valor aos seus produtos, foi apontado:

Ao longo desses 6 anos [...] os investimentos foram aumentando cada vez mais, foi agregado mais valor [...]. Saímos de um faturamento anual de um pouco menos de 4 milhões para mais de 25 milhões. Hoje [...] temos um escritório na China, em Recife e em São José dos Campos, além de uma equipe muito bem qualificada. (E2).

É possível perceber nas falas que os investimentos em P\&D agregam valor para os clientes do ITEMM, e os engenheiros ganham mais confiança e autonomia, além de se desenvolverem continuadamente. Também emergem como benefícios o aumento de receita, a contratação de funcionários, tanto pesquisadores quanto de apoio à pesquisa, e a criação de outras unidades. Como Arcuri (2016), Hasegawa e Furtado (2006) e Sperotto e Tartaruga (2017) já haviam destacado, investimentos em P\&D proporcionam às organizações maiores investimentos na produção, que repercutirão positivamente em empregos e outras rendas.

Identificou-se que, no ITEMM, ocorre a pesquisa aplicada voltada à solução de problemas com objetivos pré-definidos, englobando conhecimentos e experiências que se baseiam em variáveis advindas de um conhecimento implícito e difícil de se reproduzir, como observado por Damanpour (1996). Nesse sentido, muitas vezes os resultados dos projetos de P\&D do ITEMM podem não refletir 100\% do que tinha sido planejado pela equipe de pesquisa, ou pode-se obter um resultado diferente do proposto, mas, para os entrevistados, isso não significa que não tenha ocorrido o aproveitamento da situação, seja em absorção de novos conhecimentos, seja no desenvolvimento de uma nova tecnologia empregável em outro projeto.

Portanto, alinha-se a concepção de que os benefícios das atividades de P\&D para a organização são, independentemente do alcance da finalidade projetada, refletidos na gestão da inovação. Corroborando o pensamento de Hasegawa e Furtado (2006), E1 explana: "Existe um paradigma que pesquisa tem que dar certo na primeira tentativa, mas isso tem sido trabalhado e melhorado, fazendo com que resultados encontrados sejam aproveitados como inputs em outros projetos". Essa fala é complementada da seguinte forma: 
Existem [...] exemplos de descontinuidade, que se devem a modificações de planos de trabalhos durante o andamento ou até mesmo ao cancelamento de projetos não encerrados [...] se não houver atenção durante o caminho, muito conhecimento pode estar sendo gerado, mas pode ser totalmente desperdiçado [...] é comum se observar que, durante os trabalhos de pesquisa e desenvolvimento, podem aparecer novas perspectivas de atuação. (E3).

Indagou-se, ainda, sobre como as atividades de $\mathrm{P} \& \mathrm{D}$ podem vir a contribuir ao alcance da agregação de valor para o ITEMM e, consequentemente, às suas organizações-clientes, tendo sido obtida, para exemplificar, a seguinte afirmação:

Eu acho que o ITEMM agrega de positivo para os seus clientes e de vantagem o fato dele ser uma boa ponte entre a inovação na indústria e na academia, a gente é mais rápido na execução da inovação do que na academia [...] nós temos competências [...] flexibilidade, agilidade e foco na inovação, interesse em criar esse hub de empresas [...] a gente vai fazendo esse gerenciamento de risco do ponto de vista de alteração de rota. (E4).

Essa fala ecoa Govindarajan e Trimble (2006), que consideram relevante a distinção de produtos por meio da inovação e apontam que, através dessa diferenciação, a organização pode agregar valor para o cliente e obter sua singularidade no mercado. E2 ilustra essa percepção:

[...] somos o único ICT no Brasil focado em desenvolvimento de soluções, focado em acumulação de energia, no mundo, também não conheço nenhum outro que seja tão específico quanto a gente [...] eu não tenho concorrentes diretos, agora é claro que tem muitos institutos no Brasil que fazem projetos de pesquisas tal qual a gente em ramos parecidos que, de alguma forma, concorre conosco [...] tem mais gerenciamento de riscos vinculado ao risco de falhar na entrega para o cliente.

Conforme os entrevistados, o ITEMM cria diferentes produtos e soluções e se avulta no mercado por sua especialização, e, ainda que haja empresas atuando em linhas semelhantes, a diversidade e o conhecimento do instituto são determinantes para seu reconhecimento favorável no mercado. Diante dessas prerrogativas, destacaram-se algumas posturas relacionadas à $\mathrm{P} \& \mathrm{D}$ que podem contribuir para tornar o ITEMM um potencial gerador de inovação e valor:

Acho que o ITEMM precisa se posicionar ainda mais e se comunicar melhor por meio da inovação, nós fazemos inovação [...] é preciso se comunicar melhor nas mídias acadêmicas, a gente precisa gerar mais publicações abertas sobre as áreas de conhecimento que não são proprietários, o que que eu posso gerar documentos que vão começar a consolidar o ITEMM em nível nacional e depois internacional [...] Elevar o conhecimento dos pesquisadores [...] para que a gente, tendo a titulação, consigamos acessar outros niveis de fomento. (E4).

Ainda, para alguns entrevistados, um gap a ser superado pelo ITEMM é sua legitimação frente ao público especializado e às mídias acadêmicas, pois sua ausência nesses fóruns compromete a legitimidade do instituto. Emerge que o ITEMM precisa ir a campo, pois, ao 
atuar para resolver os vieses que lhe são colocados sem ir às empresas-clientes e se aprofundar nos seus problemas para descobrir a causa raiz, as soluções do instituto podem não as entender.

\section{Considerações finais}

Essa pesquisa abordou, indiretamente, o SNI, uma vez que o objetivo deste estudo foi analisar a influência da gestão da inovação, das atividades de P\&D, na competitividade de um instituto de pesquisa privado, com sede em diferentes unidades federativas brasileiras. Com isso em vista, ponderou-se como o ITEMM - instituto de P\&D privado que atua diretamente com inovação tecnológica (principalmente incremental) - agrega valor às tecnologias desenvolvidas, e ou transforma protótipos em bens comercializáveis, além de operacionalizar estratégias, todos aspectos com potencial para estabelecerem indicativos de seu sucesso.

O ITEMM trabalha baseando-se nas estratégias organizacionais definidas pela cúpula estratégica do seu principal cliente e mantenedor, mas não de forma engessada, tendo a chance de determinar rumos caso algum objetivo ou estratégia não se encaixe bem nas perspectivas dos pesquisadores quanto ao mercado, por exemplo. No instituto, a gestão da inovação é gerenciada como atividade estratégica, visando ao desenvolvimento organizacional.

Um aspecto favorável que aumenta a competitividade do ITEMM é que o instituto recruta profissionais para agregarem conhecimento a sua $P \& D$, o que repercute positivamente nos resultados inovativos, conforme apontado por Zanello et al. (2016). O perfil de formação dos respondentes e seu reconhecimento profissional demonstram que o ITEMM contrata buscando minimizar gaps de conhecimento, aspecto positivo na construção de competências de catching-up (Bin et al., 2015).

Os investimentos do ITEMM em P\&D são voltados para soluções tecnológicas que melhor atendam à necessidade do instituto durante o desenvolvimento de suas pesquisas. Assim, caso seja percebido que um avanço tecnológico pode vir a agregar valor e acentuar o retorno econômico, a equipe de pesquisadores pode alterar radicalmente um projeto que está sendo desenvolvido. No ITEMM, as atividades de P\&D que estão dando retorno positivo significativo, seja financeiro ou de outra ordem, se configuram em valores que retornam para serem reinvestidos no próprio instituto, oportunizando mais autonomia, mais demandas, mais conhecimento, dentre outros benefícios.

Ainda que alguns autores apontem que o tipo de P\&D desenvolvida pelo ITEMM não é suportada pelo setor privado e tem pouco retorno econômico (Albert et al., 2016), essa não é a realidade do instituto. Verificou-se que o ITEMM recebe significativo investimento, possui 
equipamentos de ponta, apresenta equipe qualificada, tem um cliente que lhe garante recursos e expertise no ramo de acumuladores de energia, e aumentou exponencialmente seu lucro no decorrer de sua atuação. Cabe destacar que, apesar das atividades de P\&D do ITEMM serem financiadas com seus próprios recursos, aspecto que, em uma análise preliminar, representa traço aparentemente positivo, Sperotto e Tartaruga (2017) advertem que, em longo prazo, essa característica pode vir a podar o desenvolvimento de projetos mais complexos do instituto.

Por ser um instituto ainda jovem, o ITEMM está buscando amadurecer seus processos internos de gestão da inovação, ressaltando-se que o instituto já trabalha com foco específico, que é o alinhamento às linhas de pesquisa e às metas estratégicas traçadas pela cúpula do seu principal cliente, ainda que outros clientes participem ativamente com novos escopos. A atuação no ambiente do cliente facilitaria o alcance desses aspectos, segundo os entrevistados.

O ITEMM tem buscado sempre ofertar às organizações-clientes inovações tecnológicas enquadradas em mais de uma área prioritária da Indústria 4.0 (Menelau et al., 2019), o que lhe posiciona na vanguarda tecnológica. Ainda assim, os entrevistados reconhecem que falta ao instituto visibilidade nas esferas de $\mathrm{P} \& \mathrm{D}$ acadêmicas, e isso deve ser buscado para trazer legitimidade ao ITEMM, consonante ao estabelecido por Findik e Beyhan (2015).

Por fim, embora a discussão aqui desenvolvida tenha se pautado na gestão da inovação do P\&D do ITEMM, enfatiza-se que o instituto tem potencial para apresentar outras experiências de pesquisa voltadas ao constructo de inovação, inclusive em uma perspectiva longitudinal. Diante do auferido, avalia-se ser uma questão de tempo para a P\&D do ITEMM se tornar, ainda mais, um potencial gerador de valor, abrindo seu portifólio para outras linhas de atuação nesse campo complexo que é o relacionado ao fomento de P\&D para inovação.

\section{Referências}

Albert, J. R. G., Yasay, D. B., \& Gaspar, R. E. (2016). Examining processes in research and development at the Department of Science and Technology (No. 2016-04). PIDS Discussion Paper Series.

Arcuri, M. (2016). Políticas de CT\&I e financiamento público à infraestrutura de C\&T: Comparações internacionais e mapeamento da infraestrutura nacional. In F. Negri \& F. H. S. Squeff (Org.), Sistemas setoriais de inovação e infraestrutura de pesquisa no Brasil (pp. 581-615). IPEA.

Baller, S., Dutta, S., \& Lanvin, B. (2016). The global information technology report 2016. World Economic Forum. http://www3.weforum.org/docs/GITR2016/WEF_GITR_Full_Report.pdf

Bardin, L. (2011). Análise de conteúdo. Edições 70. 
Bessant, J., \& Tidd, J. (2009). Inovação e empreendedorismo. Bookman.

Bin, A., Vélez, M. I., Ferro, A. F. P., Salles Filho, S. L. M., \& Mattos, C. (2015). Da P\&D à inovação: Desafios para o setor elétrico brasileiro. Gestão \& Produção, 22(3), 552564.

Bogdan, R., \& Biklen, S. (2003). Investigação qualitativa em educação: Uma introdução à teoria e aos métodos (12a ed.). Porto.

Campos, M. G., Santos, D. F. L., \& Donadon, F. A. B. (2017). Impacto dos investimentos em inovação na indústria brasileira. Revista Gestão Industrial, 13(3), 213-236.

Chesbrough, H. (2006). Open innovation: The imperative for creating and profiting from technology. Harvard Business School Press.

Conselho Nacional de Saúde [CNS]. (2016). Resolução no 510, de 07 de abril de 2016. Dispõe sobre as normas aplicáveis a pesquisas em Ciências Humanas e Sociais cujos procedimentos metodológicos envolvam a utilização de dados diretamente obtidos com os participantes.

Creswell, J. W. (2007). Projeto de pesquisa: Métodos qualitativo, quantitativo e misto (2a ed.). Artmed.

Damanpour, F. (1991). Organizational innovation: A meta-analysis of effects of determinants and moderators. Academy of Management Journal, 34(3), 555-590.

Damanpour, F. (1996). Organizational complexity and innovation: developing and testing multiple contingency models. Management Science, 42(5), 693-716.

Dosi, G. (1998). Institutions and markets in a dynamic world. The Manchester School, 56(2), 119-146.

Findik, D., \& Beyhan, B. (2015). The impact of external collaborations on firm innovation performance: Evidence from Turkey. Procedia-Social and Behavioral Sciences, 195, $1425-1434$.

Freeman, C., \& Soete, L. (1997). The economics of industrial innovation. The MIT Press. Gonçalves, C. A., Gonçalves Filho, C., \& Reis Neto, M. T. (2006). Estratégia empresarial: $O$ desafio nas organizações. Saraiva.

Govindarajan, V., \& Trimble, C. (2006). Os 10 mandamentos da inovação estratégica: Do conceito a implantação. Elsevier.

Hales, M. (2001). Birds were dinosaurs once: The diversity and evolution of research and technology organisations. RISE final report, CENTRIM Brighton.

Hasegawa, M., \& Furtado, A. T. (2006). Avaliação dos impactos de programas de P\&D. Inovação Uniemp, 2(3), 40-41. 
Instituto Brasileiro de Geografia e Estatística (IBGE) (2017). Pesquisa de inovação: PINTEC2017. https://biblioteca.ibge.gov.br/index.php/bibliotecacatalogo? view $=$ detalhes $\&$ id $=2101706$

Mazzucato, M. (2014). O Estado empreendedor: Desmascarando o mito do setor público vs. setor privado. Portfolio-Penguin.

Menelau, S., Macedo, F. G. L., Carvalho, P. L. D., Nascimento, T. G., \& Carvalho Júnior, A. D. D. (2019). Mapeamento da produção científica da Indústria 4.0 no contexto dos BRICS: Reflexões e interfaces. Cadernos EBAPE, 17(4), 1094-1114.

Morris, L. (2011). Permanent Innovation. Innovation Academy.

Organização para Cooperação e Desenvolvimento Econômico (OCDE) (1997). Manual de Oslo: Diretrizes para a coleta e interpretação de dados sobre inovação (3a ed.). OCDE/ FINEP.

Rauen, C. V. (2016). O novo marco legal da inovação no Brasil: O que muda na relação ICTempresa? Radar, 43, 21-35.

Rice, M. P., O’Connor, G. C., \& Pierantozzi, R. (2008). Implementing a learning plan to counter project uncertainty. MIT Sloan Management Review, 49(2), 54.

Rieg, D. L., \& Alves Filho, A. G. (2003). Esforço tecnológico e desempenho inovador das empresas do setor médico-hospitalar localizadas em São Carlos, SP. Gestão \& Produção, 10(3), 293-310.

Rogers, E., \& Shoemaker, F. F. (1971). Communication of innovations: A cross cultural approach. Free Press.

Schumpeter, J. (1988). A teoria do desenvolvimento econômico: Uma investigação sobre os lucros, capital, crédito, juro e o ciclo econômico. Nova Cultura.

Silva, D. O., Bagno, R. B., \& Salerno, M. S. (2014). Modelos para a gestão de inovação: Revisão e análise da literatura. Production, 24(2), 477-490.

Simantob, M., \& Lippi, R. (2003). Guia valor econômico de inovação nas empresas. Globo.

Sperotto, F. Q., \& Tartaruga, I. G. P. (2017). Inovação nas empresas industriais gaúchas: Recursos, esforços e resultados. Ind. Econ. FEE, 45(2), 47-62.

Tunes, R. H. (2015). Geografia da inovação: Território e inovação no Brasil no século XXI (Tese de Doutorado). Faculdade de Filosofia, Letras e Ciências Humanas, Universidade de São Paulo, Brasil.

Tushman, M., Anderson, P., \& O'Reilly, C. (1997). Technology cycles, innovation streams and ambidextrous organizations. In P. Anderson \& M. Tushman (Orgs.), Managing strategic innovation and change (pp. 386-414). Oxford University Press. 
Veado, J. T. (1985). O planejamento e o orçamento da atividade científica e tecnológica numa abordagem sistêmica: Um esboço preliminar. Ciência da Informação, 14(2), 73-91.

Verma, D., Mishra, A., \& Sinha, K. K. (2011). The development and application of a process model for R\&D project management in a high-tech firm: A field study. Journal of Operations Management, 29(5), 462-476.

Zanello, G., Fu, X., Mohnen, P., \& Ventresca, M. (2016). The creation and diffusion of innovation in developing countries: A systematic literature review. Journal of Economic Surveys, 30(5), 884-912. 